\title{
Multidrug resistant enteric fever in South Asia: unmet medical needs and opportunities
}

\author{
Investments in newer diagnostics and antimicrobial treatments are critical to improve management \\ of enteric fever in South Asia, say Christopher Parry and colleagues
}

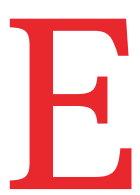

nteric fever (typhoid) is the commonest bacterial bloodstream infection in South Asia. ${ }^{1}$ It is caused by Salmonella enterica serovars Typhi and Paratyphi A. Despite progress in controlling enteric fever in several parts of the world, it remains an important public health burden in South Asia. The incidence is estimated to be over 100 per 100000 population. Around seven million people are affected each year in South Asia with about 75000 deaths. ${ }^{2}$ However, these figures are likely to be an underestimate because of limitations in population based surveillance systems and reliable diagnostic methods. ${ }^{2-4}$

We discuss the challenges in managing enteric fever in South Asia in the context of growing antimicrobial resistance and highlight the need for sustained focus on improvements in diagnosis and treatment as part of an integrated control strategy.

\section{Growing antimicrobial resistance}

Since the first reports of chloramphenicol resistance in $S$ Typhi in the 1970 s, resistance to each new antimicrobial treatment has emerged relentlessly. ${ }^{5}$ Multidrug resistance-that is, resistance to chloramphenicol, amoxicillin, and co-trimoxazole-is found in many areas of South Asia and was associated with numerous outbreaks in the late 1980 s and early 1990s.

Fluoroquinolones then emerged as the treatment of choice. ${ }^{67}$ Widespread use of second generation fluoroquinolones

\section{REY MESSAGES}

- Relentless emergence of antimicrobial resistance has led to treatment failures and limited treatment choices for typhoid fever in South Asia

- A new conjugate vaccine approved in 2018 offers an important tool to control typhoid in South Asia

- Investments in research and development of rapid diagnostic tests and new treatments must be prioritised. (ciprofloxacin and ofloxacin-levofloxacin) has now led to decreased susceptibility of organisms ${ }^{5}$ across the Indian subcontinent. ${ }^{68}$ Complete fluoroquinolone resistance, including resistance to the later generation fluoroquinolone gatifloxacin, emerged sometime after 2010 and has been associated with treatment failures and prolonged fever. ${ }^{5-10}$ The doses of fluoroquinolone used in early treatment studies, although giving a clinical cure, may have been insufficient to prevent the emergence of first step mutants.

In recent data from Pakistan published as part of the surveillance for enteric fever in Asia project (SEAP), over half of all $S$ Typhi isolates were multidrug resistant. Fluoroquinolone resistance was noted in nearly $90 \%$ of $S$ Typhi and $S$ Paratyphi isolates. ${ }^{11}$ A longitudinal study of typhoid fever trends at three large hospitals in India showed a fall in resistance rates for ampicillin, chloramphenicol, and co-trimoxazole between 2000 and 2014, as resistance to more widely used antibiotics has risen. ${ }^{12}$ Near universal resistance to ciprofloxacin has been observed in recent isolates from India. ${ }^{1213}$

Azithromycin remains an effective oral option. However, given its wide use in a variety of clinical presentations, including suspected typhoid fever and respiratory infections, there is concern about potential emergence of resistant strains. ${ }^{514}$ Third generation cephalosporins such as ceftriaxone and cefixime are increasingly used, with very low resistance reported to these drugs until recently. ${ }^{12}$ Since 2016, outbreaks of extensively drug resistant $S$ Typhi strains that are resistant to ceftriaxone and cefixime have been reported in parts of Pakistan. ${ }^{15}$ This severely limits the antimicrobial treatment options, and salvage therapy with intravenous carbapenems may be needed..$^{15}$ These drugs are expensive and often inaccessible in low resource settings.

\section{Inadequate diagnostic tools}

Typhoid presents with undifferentiated fever in the initial stages and is often confused with other common causes of acute fever in tropical regions such as malaria, dengue, chikungunya, Zika, rickettsial infection, leptospirosis, and brucellosis. ${ }^{516}$ The overlap of clinical features with other conditions makes clinical diagnosis difficult. Patients presenting with undifferentiated fever are often started on empirical treatment, once malaria has been excluded, without diagnostic confirmation. As such, diagnosis may be missed or delayed, resulting in inappropriate treatment.

Untreated, typhoid causes a prolonged and debilitating febrile illness that can last for several weeks. ${ }^{5}$ The mortality from untreated typhoid has been estimated at $10 \%$ or more. ${ }^{17}$ Patients can develop life threatening complications such as intestinal perforation, gastrointestinal bleeding, and, less commonly, encephalopathy and shock. There is also a risk of relapse and chronic faecal carriage. With appropriate antimicrobial treatment, symptoms typically resolve within a week and risk of mortality is below $1 \% .^{1819}$

Inadequate diagnostic tests complicate the management of typhoid fever. ${ }^{20}$ Isolation of $S$ Typhi on blood culture is required for a definitive diagnosis. Blood culture is also the only diagnostic method that permits monitoring for drug resistance. However, its sensitivity is suboptimal, ranging between $40 \%$ and $80 \%$. A systematic review and meta-analysis of 40 studies found a relation between specimen volume and blood culture sensitivity, with increase in sensitivity from $51 \%$ ( $2 \mathrm{~mL}$ specimen) to 65\% (10 mL specimen). Subgroup analysis showed significant heterogeneity by patient age. Previous antimicrobial use and specimen collection beyond the first week of symptoms reduced the sensitivity by nearly $30 \% .^{21}$ Clinicians' use of blood culture also varies considerably. In a prospective study from Nepal, of 4309 patients with acute fever, over half received a provisional clinical diagnosis of enteric fever, but only $4 \%$ of these patients had culture confirmed $S$ Typhi infection..$^{22}$ Blood culture requires reliable laboratory facilities and is often not feasible in low resource settings. 
In the absence of reliable and quick diagnostic tests, the Widal test is widely used by clinicians in South Asia to diagnose typhoid. ${ }^{23}$ It is a serological test that relies on a fourfold or greater rise in antibody titre against the $\mathrm{H}$ and $\mathrm{O}$ antigens of $S$ Typhi between the acute and convalescent stages of disease to confirm diagnosis and is intended as a tool to increase the index of suspicion for typhoid. Difficulties remain with the test's performance and with interpretation of results, particularly when it is used as a standalone test in the acute stage. Cross reactivity with other infectious agents $^{24}$ may result in false positive results and lead to overdiagnosis of typhoid fever. A striking example of this was noted in postearthquake Nepal, where a cross reaction of the test with rickettsia (scrub typhus) infection resulted in misdiagnosis and delay in initiating appropriate treatment. Ceftriaxone was prescribed for typhoid rather than doxycycline, which is the drug of choice for scrub typhus. ${ }^{25}$

\section{Control measures fall short}

Enteric fever is transmitted through food and water contaminated with human waste. It can be eliminated with interventions such as access to potable water, safe sanitation, and hygienic food production practices. With improvements in water quality and sanitation, countries in North America and Europe succeeded in eliminating typhoid as a public health problem.

South Asia has made considerable progress in improving access to sanitation facilities over the past 25 years. ${ }^{26}$ The proportion of people with access to improved sanitation increased from 25\% in 1990 to $48 \%$ in 2015 and the proportion of people who practise open defecation fell from $65 \%$ to $34 \%$. Three countries-Maldives, Pakistan, and Sri Lanka-have met the millennium development goals' sanitation target, with over $60 \%$ of the population having access to toilets. Bangladesh, Bhutan, and Nepal have made good progress as well. India accounts for over $60 \%$ (596 million) of the global population who practise open defecation. The Indian government launched the Swacch Bharat Mission in 2014 with the goal of reaching universal sanitation coverage by 2019 although this may be difficult to achieve. The programme has intensified efforts to raise awareness on the need for improved sanitation and safe water, construct household toilets, and systematically monitor the creation of villages and districts that have no open defecation.
Access to uncontaminated water in South Asia has increased from $73 \%$ to $93 \%$ since 1990. However, over 134 million people still do not have access to safe drinking water. Between $68 \%$ and $84 \%$ of water sources in South Asia are estimated to be contaminated with bacteria and/or chemicals. ${ }^{2627}$

Rapid unplanned urbanisation in South Asia has compounded the challenge of ensuring safe drinking water supply and access to toilets. Urban-rural disparities in access to improved water and sanitation facilities are pronounced, with rural households more commonly practising open defecation. ${ }^{28}$ Disparities in sanitation coverage are also observed between rich and poor households as well as by sex, education level, and caste. ${ }^{29}$

A small subset of patients with typhoid become chronic carriers, with faecal shedding of bacteria for several weeks after symptoms have resolved. These patients are a potential source of infection to others in the community. Despite clear links between typhoid and consumption of street foods in South Asia, there is no systematic screening for chronic carriers among food handlers. Few studies have evaluated the effectiveness of antimicrobial regimens in restricting faecal shedding.

\section{New developments in prevention, diagnosis, and treatment}

Alongside sustained efforts to improve sanitation and water safety, vaccination promises to aid typhoid control in South Asia in the coming years. Improving case management of typhoid fever requires development of alternative antimicrobial treatments and new diagnostics. Salmonella spp, particularly those that are resistant to fluoroquinolones, are among the high priority pathogens identified by WHO as requiring urgent research and new antimicrobials. ${ }^{30}$

Regional collaboration will be critical to sustain focus on typhoid elimination, boost research and development of new interventions, improve surveillance systems, and foster transfer of knowledge between nations.

\section{Vaccines}

Vaccination can have an important role in reducing the disease burden and stalling the emergence of resistant strains. Until recently, two typhoid vaccines have been available: an oral vaccine (Ty21a vaccine) supplied in enteric coated capsules taken once daily for three days, and the injectable Vi polysaccharide vaccine (ViCPS vaccine) given intramuscularly in a single dose. The protective efficacy wanes over time, and revaccination is recommended every three years. ${ }^{3132}$ Neither vaccine is recommended in children younger than 2 years, making it difficult to incorporate them into routine vaccination programmes in endemic settings, and so vaccines have been largely used in travellers to low and middle income countries.

In January 2018, WHO prequalified a conjugated Vi polysaccharide vaccine Typbar-TCV, indicating it meets the required standards of safety, efficacy, and quality to be rolled out in routine childhood immunisation programs in endemic countries. ${ }^{33}$ The vaccine, which was developed by the Indian drug company Bharat Biotech, has longer and higher levels of immunogenicity than the ViCPS vaccine and is safe to use in infants older than 6 months. In October 2017, the Strategic Advisory Group of Experts (SAGE) on immunisation recommended routine use of the vaccine in typhoid endemic countries as a single dose in children aged 6- 23 months, and for catch-up vaccination in children aged 2-15 years. ${ }^{3134}$

The vaccine has been registered in India and Nepal, but implementation of routine vaccination across South Asia will take time dependent on further approvals, availability, and funding. Post-licensing monitoring of effectiveness, persistence of protection, and safety will also be important. ${ }^{35}$ The typhoid conjugate vaccine does not protect against $S$ Paratyphi A, which is responsible for up to a fifth of cases of enteric fever. Developing a combination vaccine that provides complete protection against salmonella infections must be prioritised.

\section{Diagnostics}

Reliable, rapid diagnostics that do not require sophisticated laboratory infrastructure are needed to improve antibiotic stewardship and generate critical data on the burden of disease.

A Cochrane review in 2017 (37 studies, 5080 participants) evaluated the diagnostic accuracy of available point-of-care rapid diagnostic tests for enteric fever. ${ }^{36}$ Most studies evaluated three tests and their variants: TUBEX (14 studies); Typhidot (22 studies); and the KIT Test-It Typhoid (nine studies). These are all antibody tests on blood to detect $S$ Typhi infection. None of the included studies evaluated a test for $S$ Paratyphi A infection. Most of the studies were in South Asia: India (10 studies), Bangladesh (five), Pakistan (four). The quality of the studies was generally low, 
with two thirds including patients who would not typically be tested for this disease. The review concluded that the current tests are not sufficiently accurate to replace blood culture as a diagnostic test for enteric fever.

Newer diagnostic approaches such as serodiagnostic antigens, nucleic acid amplification tests, proteomics, metabolomics, and host response gene patterns are being explored ${ }^{20}$ but are still a considerable way from being used in routine practice. Any new tests will also need to be shown to be cost effective before they are implemented in South Asia.

\section{Effective case management}

No recent national or regional guidelines exist for treatment of enteric fever, and evidence based diagnostic pathways and empirical treatment regimens suited to South Asia are urgently needed to support clinicians.

Antimicrobial resistance patterns are highly dynamic and need to be considered carefully. Clinicians must be aware of local resistance patterns and modify treatment based on antibiotic susceptibility results in confirmed cases of enteric fever. Short fever clearance times and low relapse rates have been reported with oral azithromycin, and it may be an effective treatment. ${ }^{7}$ Lack of resistance against third generation cephalosporins, principally parenteral ceftriaxone and oral cefixime, in $S$ Typhi and $S$ Paratyphi A make these a reliable choice for suspected typhoid, particularly in regions with known resistance. For extensively drug resistant strains, potential antimicrobials include piperacillin-tazobactam, ceftazidimeavibactam, carbapenems (such as meropenem, imipenem, or ertapenem), tigecycline, fosfomycin, and colistin. These are given parenterally, but there is limited experience and evidence on their use in enteric fever.

Antimicrobial combinations are an option when the diagnosis is unclear or when polymicrobial infection is likely. For example, in areas where both typhoid and Rickettsia spp or Orientia tsutsugamushi infections are common causes of acute febrile illness, a combination of ceftriaxone and doxycycline may be appropriate as initial empirical therapy in adults, especially if confirmatory diagnostic testing is not available or while results are awaited. ${ }^{37}$ Similarly, combination treatment may be required in typhoid associated intestinal perforation to treat $S$ Typhi and other intestinal organisms causing peritonitis.
Preventing the emergence of resistance has been an important justification for the use of combination therapies in tuberculosis, HIV, and malaria. In principle, when antibiotic monotherapy is used, the drug susceptible fraction of the population is killed, while the resistant subpopulation replicates and becomes dominant. The use of two or more antimicrobials makes this less likely unless there is background resistance to both antimicrobials. For example, $S$ Typhi and $S$ Paratyphi A isolates acquire decreased susceptibility to fluoroquinolones by point mutations. Fluoroquinolone doses that are effective against the wild type isolates but fail to supress the first step mutants can select for the mutant strains. Combining a fluoroquinolone with a drug targeting a different pathway may prevent first step mutants from replacing the wild type.

Drug combinations, however, are costlier and have an increased risk of adverse events. Combining the right drugs in the appropriate doses will be critical. ${ }^{38}$ An inappropriate combination may inadvertently select for new resistance profiles. Fixed dose combinations of relevant antimicrobials are available in India, but there has been relatively little research on the pharmacokinetic and pharmacodynamic parameters that predict the successful treatment of typhoid and few clinical trials of potential combinations. ${ }^{39}$

The priorities for future research include development of new treatments; in vitro assessments of old and new drugs and drug combinations against a relevant panel of isolates, including extensively drug resistant typhoid; and clinical trials of salvage regimens for multidrug resistant enteric fever, antimicrobial combinations for suspected and confirmed enteric fever, and antimicrobial regimens to prevent chronic carriage. New treatments and diagnostics, together with better vaccines and sanitation, will be essential for elimination of typhoid in South Asia.

Contributors and sources: This article is based on a search for relevant articles in Medline, the Cochrane Collaboration, and Clinical Evidence published in English since 2008. CP prepared the first draft of the manuscript, which was reviewed and revised by all the authors. The revised version of article was prepared by IR with input and review from CP and colleagues. CP is the guarantor.

Competing interests: We have read and understood BMJ policy on declaration of interests and have no relevant interests to declare.

Provenance and peer review: Commissioned; externally peer reviewed.

This article is one of a series commissioned by The $B M J$ in collaboration with the Drugs for Neglected
Diseases initiative (DNDi). The BMJ retained full editorial control over external peer review, editing, and publication. Open access fees are funded by the DNDi, Geneva.

Christopher M Parry, consultant clinical microbiologist ${ }^{1,2}$

Isabela Ribeiro, head

Kamini Walia, senior scientist ${ }^{4}$

Priscilla Rupali, professor ${ }^{5}$

Stephen Baker, professor $r^{6,7,8}$

Buddha Basnyat, professor ${ }^{7,9}$

${ }^{1}$ Department of Clinical Sciences, Liverpool School of Tropical Medicine, Liverpool, UK

${ }^{2}$ School of Tropical Medicine and Global Health, Nagasaki University, Japan

${ }^{3}$ Dynamic Portfolio Unit, Drugs for Neglected Diseases initiative, Geneva, Switzerland

${ }^{4}$ Department of Medical Microbiology, Institute of Medical Education and Research, Chandigarh, India

${ }^{5}$ Department of Medicine, Christian Medical College, Vellore, India

${ }^{6}$ Wellcome Trust Major Overseas Programme, Oxford University Clinical Research Unit, Hospital for Tropical Diseases, Ho Chi Minh City, Vietnam

${ }^{7}$ Centre for Tropical Medicine and Global Health, Oxford University, Oxford, UK

${ }^{8}$ Department of Medicine, University of Cambridge, Cambridge, UK

${ }^{9}$ Oxford University Clinical Research Unit, Patan Academy of Health Sciences, Kathmandu, Nepal

Correspondence to: CM Parry

christopher.parry@lstmed.ac.uk

\section{(9) (1) $\Theta$ OPEN ACCESS}

This is an Open Access article distributed in accordance with the Creative Commons Attribution Non Commercial (CC BY-NC 4.0) license, which permits others to distribute, remix, adapt, build upon this work non-commercially, and license their derivative works on different terms, provided the original work is properly cited and the use is non-commercial. See http://creativecommons.org/licenses/by-nc/4.0/.

\section{Check for updates}

1 Deen J, von Seidlein L, Andersen F, Elle N, White NJ, Lubell Y. Community-acquired bacterial bloodstream infections in developing countries in south and southeast Asia: a systematic review. Lancet Infect Dis 2012;12:480-7. doi:10.1016/S14733099(12)70028-2

2 Mogasale V, Maskery B, Ochiai RL, et al. Burden of typhoid fever in low-income and middleincome countries: a systematic, literature-based update with risk-factor adjustment. Lancet Glob Health 2014;2:e570-80. doi:10.1016/S2214 109X(14)70301-8

3 Barkume C, Date K, Saha SK, et al. Phase I of the Surveillance for Enteric Fever in Asia Project (SEAP): an overview and lessons learned. J Infect Dis 2018;218(suppl_4):S188-94. doi:10.1093/ infdis/jiy522

4 Wain J, Hendriksen RS, Mikoleit ML, Keddy KH, Ochiai RL. Typhoid fever. Lancet 2015;385:1136-45. doi:10.1016/S0140-6736(13)62708-7

5 Crump JA, Sjölund-Karlsson M, Gordon MA, Parry CM. Epidemiology, clinical presentation, laboratory diagnosis, antimicrobial resistance, and antimicrobial management of invasive salmonella infections. Clin Microbiol Rev 2015;28:901-37. doi:10.1128/ CMR.00002-15 
6 Divyashree S, Nabarro LEB, Veeraraghavan B, Rupali P. Enteric fever in India: current scenario and future directions. Trop Med Int Health 2016;21:1255-62. doi:10.1111/tmi.12762

7 Trivedi NA, Shah PC. A meta-analysis comparing the safety and efficacy of azithromycin over the alternate drugs used for treatment of uncomplicated enteric fever. J Postgrad Med 2012;58:112-8. doi:10.4103/0022-3859.97172

8 Ariyal A, Basnyat B, Nhan HT, et al. Gatifloxacin versus ceftriaxone for uncomplicated enteric fever in Nepal: an open-label, two-centre, randomised controlled trial. Lancet Infect Dis 2016:16:535-45. doi:10.1016/S1473-3099(15)00530-7

9 Wong VK, Baker S, Pickard DJ, et al. Phylogeographical analysis of the dominant multidrug-resistant H58 clade of Salmonella Typhi identifies interand intracontinental transmission events. Nat Genet 2015:47:632-9. doi:10.1038/ng.3281

10 Parry CM, Vinh H, Chinh NT, et al. The influence of reduced susceptibility to fluoroquinolones in Salmonella enterica serovar Typhi on the clinical response to ofloxacin therapy. PLoS Negl Trop Dis 2011;5:e1163. doi:10.1371/journal. pntd.0001163

11 Qamar FN, Yousafzai MT, Sultana S, et al. A retrospective study of laboratory-based enteric fever surveillance, Pakistan, 2012-2014. J Infect Dis 2018;218(suppl_4):S201-5. doi:10.1093/ infdis/jiy205

12 Balaji V, Kapil A, Shastri J, et al. Longitudinal typhoid fever trends in India from 2000 to 2015. Am J Trop Med Hyg 2018;99(3_Suppl):34-40. doi:10.4269/ ajtmh.18-0139

13 Dahiya S, Sharma P, Kumari B, et al. Characterisation of antimicrobial resistance in Salmonellae during 2014-2015 from four centres across India: An ICMR antimicrobial resistance surveillance network report. Indian J Med Microbiol 2017;35:61-8. doi:10.4103/ ijmm.JJMM_16_382

14 Rai S, Jain S, Prasad KN, Ghoshal U, Dhole TN. Rationale of azithromycin prescribing practices for enteric fever in India. Indian J Med Microbiol 2012;30:30-3. doi:10.4103/0255-0857.93017

15 Klemm EJ, Shakoor S, Page AJ, et al. Emergence of an Extensively Drug-Resistant Salmonella enterica Serovar Typhi Clone Harboring a Promiscuous Plasmid Encoding Resistance to Fluoroquinolones and Third-Generation Cephalosporins. MBio 2018;9:e00105-18. doi:10.1128/ mBio.00105-18

16 Bhargava A, Ralph R, Chatterjee B, Bottieau E. Assessment and initial management of acute undifferentiated fever in tropical and subtropical regions. BMJ 2018;363:k4766. doi:10.1136/bmj. k4766

17 Buckle GC, Walker CLF, Black RE. Typhoid fever and paratyphoid fever: Systematic review to estimate global morbidity and mortality for 2010. J Glob Health 2012;2:010401. doi:10.7189/ jogh.01.010401

18 Bhutta ZA. Impact of age and drug resistance on mortality in typhoid fever. Arch Dis Child 1996;75:214-7. doi:10.1136/adc.75.3.214

19 Effa EE, Lassi ZS, Critchley JA, et al. Fluoroquinolones for treating typhoid and paratyphoid fever (enteric fever). Cochrane Database Syst Rev 2011;10:CD004530. doi:10.1002/14651858. CD004530.pub4

20 Mogasale V, Ramani E, Mogasale VV, Park J. What proportion of Salmonella Typhi cases are detected by blood culture? A systematic literature review. Ann Clin Microbiol Antimicrob 2016;15:32. doi:10.1186 s12941-016-0147-z

21 Antillon M, Saad NJ, Baker S, Pollard AJ, Pitzer VE. The relationship between blood sample volume and diagnostic sensitivity of blood culture for typhoid and paratyphoid fever: a systematic review and metaanalysis. J Infect Dis 2018;218(suppl_4):S255-67. doi:10.1093/infdis/jiy 471

22 Andrews JR, Vaidya K, Bern C, et al. High rates of enteric fever diagnosis and lower burden of culture-confirmed disease in peri-urban and rural Nepal. J Infect Dis 2018;218(suppl_4):S214-21. doi:10.1093/infdis/jix221

23 Olopoenia LA, King AL. Widal agglutination test-100 years later: still plagued by controversy. Postgrad Med J 2000;76:80-4. doi:10.1136/pmj.76.892.80

24 Parry CM, Hoa NT, Diep TS, et al. Value of a singletube widal test in diagnosis of typhoid fever in Vietnam. J Clin Microbiol 1999;37:2882-6.

25 Basnyat B. Typhoid versus typhus fever in postearthquake Nepal. Lancet Glob Health 2016;4:e5167. doi:10.1016/S2214-109X(16)30094-8

26 WHO/Unicef Joint Monitoring Programme for Water Supply and Sanitation. A snapshot of sanitation, hygiene and drinking water safety in South Asia. 2015 update. 2015. http://www.searo.who.int/ entity/water_sanitation/data/watsancoverage. pdf?ua $=1$

27 Unicef. Water, sanitation, and hygiene (WASH). https://www.unicef.org/rosa/water-sanitation-andhygiene-wash

28 Bhutta ZA, Gaffey MF, Crump JA, et al. Typhoid fever: way forward. Am I Trop Med Hyg 2018;99(3 Suppl):89-96. doi:10.4269/ajtmh.18-0111

29 WHO/Unicef Joint Monitoring Programme for Water Supply and Sanitation. JMP Update Report 2015.
2015. https://d26p6gt0m19hor.cloudfront.net/ whywater/JMP-2015-Annual-Report.pdf

30 Tacconelli E, Magrini N, Carmeli Y, et al. Global priority list of antibiotic-resistant bacteria to guide research, discovery and development of new antibiotics. World Heal Organ 2017;7. doi:10.1016/S1473-3099(09)70222-1

31 Andrews JR, Qamar FN, Charles RC, Ryan ET. Extensively drug-resistant typhoid - are conjugate vaccines arriving just in time? N Engl J Med 2018;379:1493-5. doi:10.1056/ NEJMp1803926

32 WHO. Typhoid fever https://www.who.int/ith/ vaccines/typhoidfever/en/

33 WHO. Typbar TCV from Bharat Biotech, world's first typhoid conjugate vaccine prequalified by WHO. 3 Jan 2018. https://www.who.int/medicines/news/2017/ Bharat-Biotech-TypbarTCV-WHO-PQ-Press-ReleaseGlobal-Final.pdf?ua=1

34 Andrews JR, Baker S, Marks F, et al. Typhoid conjugate vaccines: a new tool in the fight against antimicrobial resistance. Lancet Infect Dis 2018:S14733099(18)30350-5. doi:10.1016/S14733099(18)30350-5

35 World Health Organization. Meeting of the Strategic Advisory Group of Experts on immunization, October 2017-conclusions and recommendations. Wkly Epidemiol $\operatorname{Rec} 2017 ; 92: 729-47$.

36 Wijedoru L, Mallett S, Parry CM. Rapid diagnostic tests for typhoid and paratyphoid (enteric) fever. Cochrane Database Syst Rev 2017;5:CD008892. doi:10.1002/14651858.CD008892.pub2

37 Bakthavatchalam YD, Kumar DT, Tayubi IA, et al. In vitro efficacy and in silico analysis of cefixime-ofloxacin combination for Salmonella Typhi from bloodstream infection. J Appl Microbiol 2017;123:615-24. doi:10.1111/ jam.13522

38 Booker BM, Smith PF, Forrest A, et al. Application of an in vitro infection model and simulation for reevaluation of fluoroquinolone breakpoints for Salmonella enterica serotype typhi. Antimicrob Agents Chemother 2005;49:1775-81. doi:10.1128/AAC.49.5.1775-1781.2005

39 Parry CM, Ho VA, Phuong T, et al. Randomized controlled comparison of ofloxacin, azithromycin, and an ofloxacin-azithromycin combination for treatment of multidrug-resistant and nalidixic acid-resistant typhoid fever. Antimicrob Agents Chemother 2007:51:819-25. doi:10.1128/ AAC.00447-06

Cite this as: $B M J$ 2018;364:k5322

http://dx.doi.org/10.1136/bmj.k5322 